\title{
Meditation im Kontext der indischen Philosophie
}

\author{
Die Yoga-Sūtren von Patañjali
}

Die Yoga-Sütren von Patañjali stellen die erste systematische Niederschrift indischer YogaPhilosophien dar. Patañjali beschreibt darin den 8-gliedrigen Pfad des Yoga (aștāningyoga), der in der Realisation der drei inneren Glieder der yogischen Sammlung (samyama) gipfelt: Konzentration (dhāraṇā), Meditation (dhyāna) und Versenkung (samādhi). Ein Zustand, in dem nur noch der Gegenstand leuchtet. Gerade so, als ob das Ich verschwunden wäre. Für Patañjalis Auffassung von Meditation ist charakteristisch, dass dieses meditative Sein zur Welt von den Menschen zwar nicht willentlich herbeigeführt, durch die Übung der fünf äußeren Glieder von Yoga aber sehr wohl tätig vorbereitet werden kann: durch die Einübung yogischer Verhältnisse zu anderen (1), zu uns selbst (2) und die Ausbildung einer yogischen Körperhaltung (3) und Atmung (4). All diese Praktiken machen uns reif für das Ereignis jenes Sinneswandels (5), der uns das innere Selbst schmecken lässt und damit die Tore für die yogische Sammlung (6-8) öffnet.

\section{Prolog}

Das Wort „Yoga“ hat im indischen Kontext viele Bedeutungen (vgl. White 2012, S. 2). Im ältesten Veda, dem Rg Veda, der wahrscheinlich im 15. Jahrhundert vor unserer Zeitrechnung entstanden ist, bezeichnet „Yoga“ vor allem das Joch, das zwei Ochsen zu einem Gespann zusammenfügt. Aber nicht nur das Gerät, auch die Tätigkeit dieses Zusammenspannens selbst - ,yoking“, wie es noch heute im Englischen heißt - bezeichnet die Sprache des Sanskrit mit dem Wort ,Yoga“.

Durch die Verbindung der beiden Ochsen in ein Ochsengespann werden sie Bestandteile eines übergeordneten Gefüges, das die einzelnen Teile des Gespanns nicht nur äußerlich aneinander kettet, sondern zu lebendigen Aspekten einer ineinander greifenden Gerätschaft macht, die eine maschinelle Sinneinheit ergibt. Deren lebendiges Gezüge ist fortan im Stande, produktive Tätigkeiten zu vollziehen, zu denen die einzelnen Teile alleine nicht in der Lage gewesen wären. Durch die intelligente Anbindung der Lebenskraft der beiden Ochsen an die Produktionsmaschine „Ochsengespann“ werden die Teile, aus denen sich das Ensemble dieser Gerätschaft zusammensetzt, insgesamt also vermögender, kräftiger, „leistungsstärker“, „effizienter", produktiver, als sie es ohne ein solches „Yoga“ wären (vgl. Desikachar 1980, S.2).

Vermutlich kommt das, was Gilles Deleuze und Félix Guattari in Anti-Ödipus Wunschmaschinen nennen (vgl. Deleuze/Guattari 1977, S. 7-63), der ältesten Be- 
deutung von Yoga sehr nahe; bildet der semantische Herd von Wörtern wie Gerät, Gefüge, Gezüge, Gespann, Joch, Verkettung, zusammenspannen, anbinden, verbinden, einbinden, zusammensetzen, zusammenfügen, zügeln etc. in der Tat doch das semantische Feld, dem der älteste Sprachgebrauch des Wortes in den Veden entstammt.

Ohne dem Wort Gewalt anzutun, können wir Yoga im weitesten Sinne daher als eine spezifische Weise der Einigung einer Multitude verstehen: als Wie der produktiven Verfertigung eines Gefüges, das einer bestimmten Substanz die Essenz eines konkreten Ordnungsgefüges (dharma) verleiht. Ist doch überall dort, wo eine Vielheit von Aspekten durch eine stabile Verkettung in die synthetische Einheit eines dauerhaften Jochs zusammengefügt wird, im vedischen Sinne Yoga am Werk.

In diesem weiten Sinne kann z.B. Sri Aurobindo Ghose, einer der bedeutendsten indischen Dichterphilosophen des 20. Jahrhunderts, in seinem Buch The Synthesis of Yoga immer noch zwanglos von einem "evolutionary Yoga of Nature“ (Sri Aurobindo 1992, S. 22) sprechen. Die Natur selbst betreibt Yoga, und nicht nur der Mensch, wenn sie etwa ein heterogenes Gemenge atomarer Partikel in ein molekulares Ordnungsgefüge zusammenbindet, das die Essenz dieser chemischen Substanz ausmacht. Aus der Art und Weise dieses Jochs ergeben sich einerseits die physischen Eigenschaften dieses molekularen Gebildes (niyama), andererseits definiert es aber auch die Typik möglicher Wechselwirkungen und Verbindungen, in die ein solches Molekül seiner Essenz nach überhaupt treten kann (yama). Beide Momente zusammen, das Paar yama \& niyama, machen im yogischen Sinne daher erst die Natur (dharma) einer Sache aus. Ihr Ethos, im etymologischen Sinne des altgriechischen Wortes ,ethos“, das ursprünglich den Aufenthaltsort einer lokalen Erscheinung inmitten des Seienden im Ganzen charakterisierte; also die kosmische Position, die ein Einzelding inmitten der Welt einnimmt. Das so verstandene Ethos eines Dings reguliert nicht nur die möglichen räumlichen Beziehungen eines Körpers zu anderen Körpern, die ihm seiner Natur (dharma) nach gesetzmäßig zukommen. Es definiert auch den Vermögensgrad, den eine Substanz inmitten aller anderen Substanzen rangmäßig innehat - ihre kosmische Stellung und Würde, die sie in Bezug auf alle anderen Körper, mit denen sie ihr In-der-Welt-sein ${ }^{1}$ teilt, naturgemäß einnimmt.

Zum Ethos des Menschen gehören im Kontext eines solchen Sprachgebrauchs daher nicht nur die sozialen Beziehungen, die Menschen miteinander unterhalten. Auch die eigentümlichen Verschränkungen der menschlichen Natur mit nichtmenschlichen Dingen sind Teil des menschlichen Ethos. Angefangen von seiner leiblichen Verschränkung mit der anorganischen Natur, über seine lebensweltliche Verschränkung mit anderen Lebewesen wie Pflanzen und Tieren, bis hin zu den Artefakten, die von Menschen selbst hervorgebracht werden und seine Lebenswelt ganz maßgeblich mitbestimmen.

Hinter der abstrakt anmutenden Frage nach der Essenz einer bestimmten Substanz steht im Kontext indischer Philosophien daher ein ganz konkretes „ethisches“

1 Schreibweise nach Heidegger. 
Problem. Die Frage, die auch Spinoza in seiner Ethik wiederholt stellt, „was der Körper kann“ (Spinoza 2007, S. 229, 231). Sie zielt auf die konkrete Klärung des Vermögensgrads eines Körpers ab, der sein Ethos ausmacht. Entscheidet das, was ein Körper kann, doch darüber, welche Wechselwirkungen mit anderen Körpern dem Erhalt und Wachstum eines Körpers (conatus) förderlich bzw. hinderlich sind, klișta bzw. aklișța, wie Yoga-Sūtra 1.5 sagt (vgl. Bäumer 2007, S. 28).

Je nachdem, ob die Vermengung eines Körpers mit anderen Körpern den Vermögensgrad der involvierten Substanzen steigert oder hemmt, kräftigt oder schwächt, freudig stimuliert oder ,gehässig“ vergiftet, am Leben erhält und wachsen lässt oder gar zerstört und vernichtet, werden diese beiden Körper einander auch in ,ethischer“ Hinsicht gut oder schlecht nennen.

\section{Der acht-gliedrige Yogaweg zur Versenkung}

Dieser Hinweis auf die philosophische Grundbedeutung der beiden Termini yama \& niyama ist für eine Erörterung der Stellung von Meditation im Kontext der Yoga-Sūtren von Patañjali wesentlich, da Patañjali seinen acht-gliedrigen Yogaweg (aștâñga-yoga) ausdrücklich mit den beiden Gliedern yama \& niyama beginnen lässt (vgl. Bäumer 2007, YS 2.29, S. 115). Dabei unterscheidet er in seiner SūtrenSammlung, die vermutlich im 4. Jahrhundert entstanden ist und damit die älteste schriftliche Überlieferung einer systematischen Textsammlung yogischer Praktiken darstellt, zwischen fünf äußeren und drei inneren Gliedern. Zusammen ergeben sie, wie das Wort aștāng ga sagt, den achtgliedrigen Pfad seines Yoga-Weges.

Die ersten fünf (äußeren) Glieder zielen darauf ab, uns auf die yogische Sammlung vorzubereiten. Sie bringen uns auf den Yoga-Weg, indem sie uns aus unserer zerstreuten Seinsverfassung zurückholen. Während es in diesen Gliedern des Yogaweges darum geht, eine yogische Seinsverfassung erst zu erwerben, beschreiben die drei letzten inneren Glieder von Patañjalis aștānga-yoga das Ereignis dieser yogischen Sammlung selbst. Sie realisiert sich im Zuge einer dreistufigen Erfahrung - dhāraṇa (Konzentration), dhyāna (Meditation) und samādhi (Versenkung) -, deren Trias den Sammelnamen samyama trägt (vgl. Bäumer 2007, YS 3.4, S. 134).

Wenn wir die Eigentümlichkeit des yogischen Meditationsweges erfassen wollen, dann müssen wir im Folgenden alle acht Stufen von Patañjalis aștāñga-yoga im Auge behalten. Denn weder lässt sich der Meditationsweg von Yoga auf das Ziel, die yogische Sammlung (samyama), reduzieren noch auf irgendwelche äußeren Glieder, etwa auf die Übung von Körperstellungen (āsana) oder Atemtechniken (prānāàāma).

Auch wenn die fünf ersten Glieder „bloß“ eine äußere, vorbereitende Funktion für die Realisation der drei letzten Glieder haben, beginnt der yogische Meditationsweg nicht mit den drei Meditationspraktiken im engeren Sinne, sondern mit den beiden ersten äußeren Gliedern yama \& niyama: 
(1) yama, das erste, grund-legende Glied von aștāinga-yoga, umfasst das Gelübde der fünf äußeren ethischen Selbstdisziplinierungen gegenüber allen anderen Dingen und Lebewesen. Durch die Praxis von yama soll ein yogisches Beziehungsgeflecht zu anderen generiert werden, das eine weltweite Erfahrung von samädhi inmitten der Natur ermöglichen soll.

(2) niyama, das zweite Glied von așțànga-yoga, umfasst hingegen die fünf inneren Selbstdisziplinierungen gegenüber uns selbst. Durch die Praxis von niyama soll die leibhaftige Sammlung unserer eigenen lokalen Existenz in samādhi inmitten der Natur ermöglicht werden.

Wenn wir den Meditationsweg der Yoga-Sūtren also tatsächlich mit den beiden ersten Gliedern von Patañjalis aștāngna-yoga beginnen lassen, dann wird klar, dass Meditation im Kontext der Yoga-Sūtren nicht einfach heißt, dass sich ein privates Subjekt in eine Wüste oder ein Zimmer zurückzieht, um dort „in sich“ zu gehen und zu „meditieren“. Wie YS 1.2 und 1.4 gleich eingangs vermerken, ist nämlich die Lage des Subjekts, an das sich die Yoga-Sūtren wenden, durch und durch zwiespältig. Einerseits definiert YS 1.2 (vgl. Bäumer 2007, S. 21) Yoga, in einer berühmten Formulierung, als ,yogaś-citta-vrtti-nirodhah" ${ }^{\text {“ }}$ als eine leibhaftige Selbsterfahrung (citta), in der die Wirbel der Zeit (vrtti) zur Ruhe kommen (nirodha). Andererseits wird dieser yogischen Seinsverfassung in YS 1.4 (vgl. Bäumer 2007, S.21) aber umgehend die menschlich-allzumenschliche Verfassung eines zerstreuten Subjekts entgegengestellt, das sich fälschlicherweise mit den Wirbeln der Zeit identifiziert (vrtti-sārüpya) und daher in eine fatale, weil Leid erzeugende Seinsverfassung gerät. Da eine solche Disposition die yogische Entfaltung im Menschen hemmt, steht sie dieser sogar diametral entgegen (itaratra).

Um dieser zwiespältigen Lage zu entkommen und die zerstreute Seinsverfassung des Menschen in eine yogische zu transformieren, stützt sich der Meditationsweg von aștânga-yoga neben (1) yama und (2) niyama noch auf drei weitere äußere Glieder:

(3) āsana, Körperstellungen. In diesem dritten Glied wird eine komfortable, stabile, entspannte und dem Unendlichen zugewandte yogische Körperhaltung geübt. Hat der Organismus die eingeübte Körperhaltung einmal habitualisiert, sprich leibhaftig an-genommen, dann ist sie für ihn, wie wir in Anklang an Deleuze und Guattari sagen könnten, maschinell abrufbar geworden. Unser Leib vermag nun quasi ganz von selbst auf sie zurückzugreifen - automatisch, reflexartig, unbewusst, unwillkürlich, instinktiv, „organisch“, „,natürlich“, ,habituell“, um sie am eigenen Leib zu reproduzieren, wann immer sie ihm geboten scheint.

(4) prānāyāma, Atemtechniken. Sie bilden das vierte Glied des achtgliedrigen Yogaweges. In dieser yogischen Praxis geht es um die Ausbildung einer yogischen Atmung, in der wir uns des Einatmens, des Ausatmens, der Atempausen, der Atemlänge, der Subtilität der eigenen Atemqualität, der leiblichen Verortung unserer Atmung, ihrer Dauer und der Anzahl der Atemzüge beim Atmen bewusst werden. Vor allem die Verlängerung der Atempausen in den Atemkehren zwischen Einund Ausatmung sowie Aus- und Einatmung erlauben es uns, den Moment des 
Atemstillstands zu gewahren und uns so mit dem Totpunkt der Atmung schon im Leben anzufreunden.

(5) pratyāhāra, die Umwendung der Sinne. Sie markiert das fünfte und letzte der fünf äußeren Glieder von Patañjalis aștāngna-yoga. Auf dieser Stufe des Yogawegs findet die Umkehr der gewöhnlichen Blickrichtung der Sinne statt. Entgegen ihrer natürlichen Funktion, in der sie uns in ein offenständiges Verhältnis zu äußeren Gegenständen versetzen, bekommen wir im Zuge ihrer Rückbindung an die „innere“ Form der Leerheit (svarūpa-śünyam; vgl. YS 2.54, YS 3.3) einen ersten Geschmack vom „Nichts“ zu schmecken; von jenem „eigenschaftslosen Seyn“" (śūnyānām-gunānām, vgl. YS 4.34), das dem ewigen Werden der Natur (prakrti) sonst meist unbemerkt zugrunde liegt, auch wenn wir es im Grunde immer schon selbst sind.

Dieser unvermutete Umschlag der Sinnrichtung der Sinne, der in pratyāhāra stattfindet, ist für Patañjali jenes Ereignis, das in einem ganz besonderen Maße dafür geeignet ist, uns auf die Erfahrung der drei inneren Glieder der yogischen Sammlung einzustimmen und damit in den inneren Kern des yogischen Meditationswegs vordringen zu lassen.

Nicht zufällig endet das zweite Kapitel der Yoga-Sūtren, das den Pfad der Übung (Sādhana Pāda) beschreibt, mit der Beschreibung dieses außergewöhnlichen Sinnenwandels, der in pratyāhāra Ereignis wird; gehören doch die drei letzten Glieder des acht-gliedrigen Yogaweges, kraft ihrer außerordentlichen Leistungsfähigkeit, nicht mehr dem Pfad der Übung, sondern dem Pfad „übernatürlicher“ Kräfte (vibhūti pāda) an, der erst im dritten Kapitel der Yoga-Sūtren besprochen wird.

Dass die letzten drei Glieder des acht Glieder umfassenden aștānga-yoga nicht mehr im zweiten, sondern erst zu Beginn des dritten Kapitels behandelt werden, muss nicht zwangsläufig als ein „Fehler“ des Autors gedeutet werden, der angeblich die These unterstreicht, dass dieser Text zu unterschiedlichen Zeiten verfasst wurde. Könnte dieser merkwürdige Sachverhalt doch auch als formaler Hinweis darauf gelesen werden, dass das Ereignis der meditativen Sammlung eines Menschen von einem Menschen im eigentlichen Sinne des Wortes gerade nicht mehr „geübt" werden kann, da die Trias der yogischen Sammlung nichts ist, was durch das Üben irgendeiner yogischen Technik vom Menschen einfach hergestellt werden könnte.

Dass diese Lesart nicht nur formal, sondern auch inhaltlich mit dem Text der Yoga-Sütren übereinstimmt, wird vor allem im vierten und letzten Kapitel der Yoga-Sūtren deutlich. Wenn YS 4.2 ausdrücklich darauf hinweist, dass die Verwandlung einer Gattung in eine neuartige Natur nicht durch den Menschen bewirkt werden kann, sondern ,aufgrund des Überströmens der Urnatur“ (Bäumer 2007, S. 163) geschieht, dann heißt das eben, dass die Reifung der Natur (prakrtyāpüra) nicht dem Willen des Menschen und seinen (technischen) Machenschaften folgt, sondern der Wille eines Menschen umgekehrt dem Reifegrad seiner Natur. Dieser yogische Sachverhalt, der den unbedingten Narzissmus des menschlichen Willens

2 Schreibweise nach Heidegger. 
und die Leistungsfähigkeit aller Technik in Bezug auf die Realisation der höchsten „spirituellen“ Ziele in Schranken weist, wird in dem nachfolgenden Sūtra 4.3 noch einmal unterstrichen, wenn gesagt wird, dass die Kausalität des menschlichen Willens die Vorgänge der Natur nur kultivieren, aber nicht bewirken könne (vgl. Bäumer 2007, S. 163). Gleich einem Bauer, der die Dämme an seinem Reisfeld niederreißt, um das Wasser in die Felder zu fluten, vermag ein Mensch die spirituellen Hindernisse (kleśa) auf dem Yoga-Weg zwar tatkräftig zu dekonstruieren, abzubauen und schließlich niederzureißen; die überströmende Natur selbst aber, die sich dadurch ungehemmter ihren (spirituellen) Weg in einem Menschen bahnen kann, hat nach yogischer Ansicht nicht der Mensch selbst bewirkt. Er wird vielmehr, kraft seiner Geburt inmitten der Natur, in eine solche überströmende Natur geworfen.

So gesehen bricht das Ereignis der Trias der meditativen Sammlung (samyama) in der Tat als ein ,übernatürliches“ Ereignis über einen Menschen herein. Denn erst dann, wenn die Natur selbst alle kosmischen Bedingungen gezeitigt hat, deren Zusammenkunft die reife Frucht einer yogischen Sammlung zulassen, vermag uns diese Potenz der Natur, wenn überhaupt, de facto heimzusuchen. Nirgendwo sonst ist der Ausdruck Spinozas „Gott sive Natur“ (vgl. Spinoza 2007, S. 389) angebrachter als im Kontext einer philosophischen Betrachtung der Yoga-Sūtren.

\section{Kriyā-Yoga, der Yoga der Tat}

Die Kultur des Menschen und seine Techniken können das ewige Werden der Natur (prakrti) nach Patañjali daher immer nur fördern, stimulieren und beschleunigen, oder aber hemmen, deprimieren und verzögern.

Das gilt auch für die Praxis von kriyā-yoga, dem Yoga der Tat, der im zweiten Kapitel der Yoga-Sūtren besprochen wird und die Frage behandelt, was ein „Subjekt" im Kontext von aștāinga-yoga in der Tat selbst tun kann; oder genauer gesagt, zulassen sollte, wenn es sich tatkräftig dazu entschließt, sich selbst auf den Weg des Yoga zu begeben.

Die Antwort, die YS 2.2 auf diese Frage gibt, ist schlicht. Einerseits geht es beim Yoga der Tat darum, das Samādhi-Werden (samādhi-bhāvanā) in einem Menschen zu fördern, andererseits geht es kriya -yoga darum, die hemmenden Kräfte (kleśa), die einer solchen zweiten, yogischen Geburt im Menschen im Wege stehen, zu reduzieren $(\tan \bar{u})$ (vgl. Bäumer 2007, S. 83). Durch den tatkräftigen Vollzug von Yoga sollen die Dämme der blockierenden Kräfte, die die „spirituelle“ Entwicklung der Natur im Menschen hemmen, schließlich also niedergerissen und das Wachstum der meditativen Versenkung (samādhi) vorangetrieben und beschleunigt werden. Es geht mithin um einen Prozess, in dem die Gegen-Strömung jener yogischen Freiheit (pratiprasava-kaivalya) in Fluss gebracht wird, die einen Menschen aus seiner Zerstreuung zurückholt und den namenlosen Grund allen Werdens schmecken lässt. Dieser yogische „Fluss der Wahrheit“, der die Natur überfließen lässt (prakrtyāpūra), ist für Patañjali aber, wie gesagt, gerade keine menschliche Konstruktion. Er ist vielmehr eine, der Natur selbst immanente, kosmische Sinn- 
ausrichtung, in der die Natur ihre eigene (spirituelle) Natur inmitten der Natur selbst physisch zur Entfaltung bringt (vgl. Bäumer 2007, YS 2.18, S. 106).

Diese besondere Ent-Faltungsmöglichkeit der Natur wird inmitten der Natur immer dann Ereignis, wenn die Trias der yogischen Sammlung (samyama) einen Menschen überkommt, und die Natur damit ihren eigenen yogischen Selbstzweck inmitten der Natur leibhaftig erfüllt.

\section{Die yogische Sammlung (samyama)}

Die Yoga-Sūtren beschreiben die drei inneren Glieder einer solchen yogischen Sammlung der Natur im Menschen wie folgt:

(6) dhāranāa, Konzentration. Sie ist das sechste Glied von aștāinga-yoga. In YS 3.1 wird sie als die Fähigkeit eines Selbst definiert, sich in Verbindung mit einem bestimmten Gegenstand in der Leere des Raumes halten zu können (vgl. Bäumer 2007, S. 123). Die intentionale Ablenkung und Zerstreuung des Selbst von anderen Objekten verringert sich dabei umso mehr, je größer die Fähigkeit des Selbst wird, sich ausschließlich auf einen einzigen Gegenstand intentional konzentrieren zu lernen.

(7) dhyāna, Meditation. Sie ist das siebte Glied von aștāinga-yoga. In YS 3.2 wird sie als ein Modus der Sammlung bezeichnet, in dem die Konzentration auf einen Gegenstand mit dem Akt seiner Wahrnehmung völlig eins wird. Das vernehmende Subjekt und das vernommene Objekt bilden jetzt zusammen ein einziges Joch, das beide zu einem in sich schwingenden und in sich stimmigen Subjekt-Objekt-Gefüge zusammenspannt (vgl. Bäumer 2007, S. 123).

(8) samādhi, die Versenkung. Sie ist das letzte und achte Glied von aștânigayoga. In YS 3.3 wird sie als ein Zustand der Sammlung beschrieben, in der es so scheint, als ob (iva) das Ich verschwunden wäre (svarüpa-śūnyam) und nur noch der Gegenstand selbst leuchten würde (vgl. Bäumer 2007, S. 123). Ganz so, als hätte sich das kosmische Selbst nun seiner ignoranten Selbstzentrierung im Nullpunkt einer leiblichen Ichperspektive gänzlich entledigt und die unheilvolle Identifikation von Ich und Selbst ein für alle Mal hinter sich gelassen.

Samādhi, das letzte Glied von aștāñga-yoga, in dem sich der acht-gliedrige Meditationsweg von Patañjali vollendet und in eine einzigartige Erfahrung versammelt, weist damit exakt jene Eigenschaften auf, in denen wir uns von der Disposition der fünf Leid erzeugenden Spannungen befreit haben, die das Samädhi-Werden eines Menschen hemmen. Zu diesen yogischen Hindernissen zählt YS 2.3 die Unwissenheit (avidyā), Ichverhaftung (asmitā), Gier (rāga), Hass (dveșa) und Angst (abhiniveśa).

Avidyā, die Unwissenheit, ist der Nährboden aller anderen vier leidvollen Spannungen (kleśa). Unter ihrem Regime fallen wir einer kosmischen Verblendung anheim (avidyā-khyāti), die, wie YS 2.5 präzisiert, das „Vergängliche für unvergänglich, das Reine für unrein, das Leidvolle für Freude und das Nicht-Selbst für das Selbst hält““ (Bäumer 2007, S. 89). 
Asmitā, der zweite Leid erzeugende Faktor, der die Geburt einer yogischen Seinsverfassung hemmt, besteht in jener falschen Ichzentrierung, in der sich die Kraft des sehenden Subjekts fälschlicherweise mit dem Gesehenen identifiziert und von diesem nicht mehr zu unterscheiden weiß (vgl. Bäumer 2007, YS 2.6, S. 89).

Răga, die aus Ignoranz entwachsene Gier, ist der dritte Ast Leid erzeugender Kräfte; sie lässt uns aufgrund unserer ignoranten Ich-Zentrierung falschen Glücksversprechen nachjagen, die uns Glück verheißen und doch ins Unglück stürzen (vgl. Bäumer 2007, YS 2.7, S. 89).

Dvesa, das Ressentiment, bildet den vierten Ast Leid erzeugender Faktoren; lässt es uns umgekehrt doch unbegründeten Aversionen nachhängen, die uns glückliche Umstände abwehren lassen, obwohl sie uns förderlich wären (vgl. Bäumer 2007, YS 2.8, S. 89).

Abhiniveśa, der fünfte und letzte Arm Leid erzeugender Spannungen, ist die Angst, die sich aus dem unbedingten Selbstbehauptungswillen der eigenen ignoranten Selbstzentrierung ergibt und von Hass und Gier ständig genährt wird (vgl. Bäumer 2007, YS 2.9, S.90). Von ihrem unbedingten Selbstbehauptungswillen sind selbst die Weisen nie ganz frei. Sie erweist sich daher als das hartnäckigste Hindernis auf dem Weg zu samādhi.

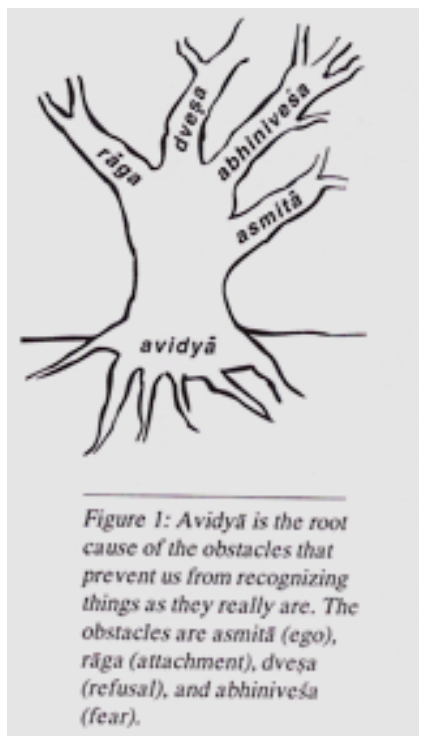

Abb. 1: Kleśa-Rhizom mit den fünf „Feinden“ des Yoga (aus: T.K.V. Desikachar: The Heart of Yoga. Developing a Personal Practice. 2. Aufl. Rochester 1999, S. 11).

\section{Das yogische Glück im menschlichen Unglück: viveka-khyāti}

Nachhaltig kann diese fatale Disposition, in der wir „Sein und Werden“, „Selbst und Nichtselbst", „Glück und Unglück“, „Wesen und Nichtwesen“, „Sehendes und Gesehenes“ etc. irrtümlich verwechseln, nach yogischer Ansicht nur durch die Her- 
vorbringung einer ganz anders gearteten ,intellektualen Anschauung“ überwunden werden, in der wir das Unterscheidungsvermögen (viveka) zwischen diesen Bestimmungen generieren.

Diese der avidyā-khyāti entgegen gesetzte yogische Schau nennen die YogaSūtren viveka-khyāti. Sie ist die (kosmische) Weisheit (prajñā), die den Yogi überkommt, wenn er von den beiden ersten inneren Gliedern der yogischen Sammlung - Konzentration und Meditation - ergriffen wird. Daher erstreckt sich die Macht der Weisheit, ,die aus dieser (unterscheidenden Erkenntnis) entspringt, [...] auf sieben Stufen (des achtgliedrigen Yoga)“ (Bäumer 2007, YS 2.27, S. 115). Erlaubt diese yogische Intelligenz dem Weisen (vivekin) doch zum ersten Mal, den konstitutiven Unterschied der beiden Prinzipien SELBST (purușa) und NATUR (prakrti) am eigenen Leib einzusehen und schließlich auch in der Natur insgesamt unterscheiden zu lernen. Wie YS 2.28 sagt (vgl. Bäumer 2007, S. 115), kann diese yogische Weisheit aber erst aufleuchten, wenn die Leid erzeugenden Spannungen (kleśa-karma) durch die Übung von aștänga-yoga schon weitgehend aufgehoben wurden.

Viveka-khyāti, die Fähigkeit, zwischen dem (geistigen) SELBST und der materiellen NATUR unterscheiden zu können, enthüllt sich für Patañjali daher als das einzig probate gnostische (jñ̄ana) Mittel, das Regime des Nichtwissens, samt seinen vier Ästen, nachhaltig zu entthronen und langfristig außer Kraft zu setzen (vgl. Bäumer 2007, YS 2.26, S. 115).

Die beiden vorletzten Glieder von aștānga-yoga, Konzentration (dhāranāa) und Meditation (dhyāna) zielen daher vor allem auf die Hervorbringung dieser (kosmischen) Unterscheidungsfähigkeit (viveka) im Menschen ab. Yogische Meditation soll die spirituelle Urteilskraft im Menschen also schärfen und nicht mindern oder gar außer Kraft setzen.

\section{Die Yoga-Sūtren von Patañajli als eine Textsammlung vorphilosophischer Praktiken}

Auch wenn die Yoga-Sūtren von Patañjali eine schon lange vor ihrer Niederschrift bestehende Yoga-Tradition in Indien „nur“ zusammengefasst und systematisiert haben, so stellt diese Sammlung von 195 Merksätzen doch das älteste schriftlich überlieferte System von Yoga dar. Dass es auf vorphilosophischen Praktiken und verschiedensten philosophischen Strömungen seiner Zeit beruht, allen voran der Sāṃkhya-Philosophie, aber auch einer Vielzahl buddhistischer Gedanken, die vor allem im ersten Kapitel in die Sūtren-Sammlung Eingang gefunden haben, sollte nicht von vornherein als Mangel gelesen werden. Denn offenkundig entspricht ein solches Vorgehen nicht nur inhaltlich, sondern auch formal der eingangs erwähnten weiten Definition von Yoga als Sammlung einer Multitude.

Im Falle einer Textsammlung wie den Yoga-Sūtren beinhaltet Yoga offenkundig auch eine schriftliche Sammeltätigkeit, in der eine heterogene Gemengelage unterschiedlichster philosophischer Texte und mündlich tradierter Yoga-Praktiken 
in ein konsistentes Textgefüge zusammentragen wird, das ihnen ein einheitliches und systematisches Gepräge gibt.

Dass sich die Yoga-Sūtren offenkundig selbst als eine solche Textsammlung verstehen, die nicht den Anspruch erhebt, das Ergebnis eines einmaligen Offenbarungsgeschehens zu sein, in dem sich der ,ewige Sinn von Yoga“ in einer unüberbietbaren Erleuchtungserfahrung des Autors ein für alle Mal unumstößlich ereignet haben soll, stellt, zumindest im Kontext einer poststrukturalistischen Lektüre der Yoga-Sūtren, gerade eine Auszeichnung dieser Schrift dar und keinen Mangel. Zeigt es doch nur, dass hier noch der alte ursprüngliche Sinn von yogischer Sammlung am Werk ist: die Zusammenfügung einer Multitude differentieller Elemente in ein stabiles, in sich stimmiges und daher auch komfortables Joch. Selbst wenn bei der Herstellung dieser Textsammlung ein Ensemble von mehreren Autoren am Werk gewesen sein sollte, dann stellt sich die Frage, warum die Einsicht, dass dieser Text das Ergebnis einer Ensemblearbeit gewesen ist, die „Autorität“ des Textes verringern und nicht vergrößern sollte.

\section{Zur Kosmologie der Sāṃkhya-Yoga-Philosophie}

Sāmkhya und Yoga werden klassisch als dualistische Philosophien charakterisiert. Diese Feststellung ist für ein adäquates Verständnis von Meditation im Kontext von Yoga von grundlegender Bedeutung; geht doch eine dualistische Philosophie, im Unterschied zu monistischen Emanationslehren, in denen die Differenzen und Vielheiten dem Einen per se untergeordnet werden, per se von einer unableitbaren Mehrheit heterogener Aspekte und Prinzipien aus - von mindestens zwei -, die auf unterschiedlichste Art und Weise vereint werden können, um dabei ein einheitliches Joch zu bilden.

Die beiden letzten, aufeinander unreduzierbaren, weil von nichts anderem mehr ableitbaren und doch ewig aufeinander verwiesenen kosmischen Prinzipien, aus deren Zusammenfügung die modale Vielheit der Formen der Welt entspringt, sind nach Sāmkhya-Yoga-Lehre die beiden Prinzipen SELBST (puruṣa) und MATERIE (prakrti).

Purușa, das (kosmische) SELBST, wird dabei als ewiges, in-sich-ruhendes Prinzip gedacht, dem sich das Werden der Welt nur insofern verdankt, als es von ihm zugelassen wird. Es besitzt folglich einen rein passiven, sein-lassenden Charakter. Im Unterschied zur materiellen NATUR (prakrti) ist es ein Prinzip, das sich selbst gelichtet ist, wodurch es sich selbst vernehmen kann. Phänomenal erfahren wir das daran, dass es in Kontakt (samyoga) mit der NATUR (prakrti) Formen leibhaftiger Selbsterfahrungen (Tautegorien) hervorbringt. In diesen erleidet es selbst eine Unmenge von Modifikationen, während es losgelöst von seiner Verbindung (samyoga) mit der materiellen NATUR gänzlich unveränderlich in sich selbst ruht und keinerlei Modifikationen unterworfen ist.

Prakrti, das kosmische Prinzip der materiellen Natur ist hingegen ein ewig zeugendes, die materiellen Formen der Welt aktiv hervorbringendes Prinzip. Im Zuge 
ihres unendlichen Werdens durchläuft sie selbst unendlich viele Modifikationen, in denen sie sich auf modale Art und Weise innerhalb der Natur materiell zum Ausdruck bringt. Im Unterschied zum SELBST vermag die NATUR ihre eigene Aktivität aber gerade nicht selbst wahrzunehmen. Sie fabriziert zwar unentwegt Substanzen in der Natur, aber unbewusst. Bewusst kann ihr schöpferisches Walten nur vom puruṣa registriert werden.

\section{Die Ambivalenz unserer leiblichen Natur}

Auch wenn die NATUR (prakrti) nur vom SELBST (purușa) erkannt werden kann, so gibt sie ihm, dem kosmischen SELBST, doch die materiellen Mittel an die Hand, die es ihm erlauben, nicht nur die NATUR, sondern auch sich selbst inmitten der Natur gewahren zu können. Denn auch wenn das SELBST losgelöst von der Natur völlig abgeschieden rein in sich selbst zu ruhen vermag, so vermag es das, was immer es vernimmt, wie YS 2.20 sagt, doch nur im Zuge einer sinnlichen Erfahrung (pratyaya), also leibhaftig wahrzunehmen (vgl. Bäumer 2007, S. 106). Da sich eine Ich-Form (asmitā, ahamkāara) im Kontext der Sāmkhya-Yoga-Lehren erst durch samyoga, die konkrete Verbindung von SELBST \& MATERIE in einem Leib (citta) ausbildet, kann sich das SELBST daher auch einzig und allein im Zuge seiner leiblichen Selbst-Objektivation inmitten der NATUR seiner selbst inne und gewahr werden.

In Hinblick auf diese ausgezeichnete Möglichkeit (vgl. Bäumer 2007, YS 2.18, S. 106) erscheint die materielle NATUR als der alleinige Ort, der die erlösende Selbsterkenntnis des (kosmischen) SELBST (samādhi) ermöglicht.

Da den Menschen das Ereignis von samādhi im Zuge ihrer leibhaftigen Geburt inmitten der Natur in der Regel aber nicht einfach in die Wiege gelegt worden ist, sondern ihrer Zerstreutheit (vrtti-sārūpya) und ignoranten (avidyā-khyāti) Seinsverfassung im Durchgang durch die Zeit dialektisch erst abgerungen werden muss, ist die leibliche Geburt eines Menschen inmitten der Natur, yogisch gesehen, durch und durch ambivalent.

Gerade diese zwiespältige Natur macht im Kontext der Yoga-Sūtren den qualitativen Unterschied der menschlichen und göttlichen Natur aus. Denn İ́vara, dem Gott der Yoga-Sūtren, kommt die Kraft der yogischen Sammlung nicht erst im Durchgang durch die Zeit (akzidentiell) zu, sondern von Ewigkeit her. Als konstitutives Wesensprädikat seiner göttlichen Seinsverfassung gehört sie ihm per se zu (vgl. Bäumer 2007, YS 2.25, S. 48). Da seinem samādhi also nie eine Zeit der Unwissenheit (avidya $)$ vorausgegangen ist, aus der er sich das göttliche Attribut der yogischen Sammlung erst erwerben hätte müssen, sind seine Handlungen (karma) seit jeher frei von Leid erzeugenden Kräften (kleśa), ihren Folgen (vipāka) und den traumatischen Sedimenten (äśayair), die ihre peinigenden Spuren weltweit hinterlassen (vgl. Bäumer 2007, YS 2.24, S.48). 


\section{Die frohe Botschaft}

Ein Mensch muss sich hingegen in der Regel vom Diktat der fünf Leid erzeugenden Faktoren durch die „yogische Schau“ von viveka-khyäti erst emanzipieren. Dass solches überhaupt möglich ist, ist sozusagen die gute Nachricht, die Yoga den Menschen übermittelt. Denn weder besteht die Natur des Menschen sub specie aeternitatis darin, dass ein Mensch ein für alle Mal in den Fängen der Knechtschaft der Un-Wissenheit gefangen bleiben muss, noch erschöpft sich das Vermögen der NATUR (prakrti) nach yogischer Ansicht darin, dass SIE im Zuge ihres ewigen Werdegangs an dem Versuch der Überwindung eines solchen leidvollen Dispositivs auf tragische Art und Weise ewig scheitern müsste.

Im Unterschied zur Konzeption einer tragischen oder sündigen Natur des Menschen geht der Meditationsweg von Yoga vielmehr von einer tief greifenden Verwandlungsmöglichkeit (parināma) der ignoranten Natur des Menschen aus. Wie Bauern die Kraft zweier Ochsen in das Joch eines stabilen Ochsengespanns bündeln können, so können nach yogischer Lehre die beiden „kosmischen Ochsen“ SELBST (purusa) und MATERIE (prakrti) durch die Mittel und Techniken von aștāinga-yoga in ein stabiles und komfortables spirituelles „Joch“ zusammengefügt werden.

In einer solchen Sammlung erfüllt sich nicht nur die Würde des Menschen, sondern auch die Würde der NATUR, in der SIE bezeugt, dass SIE in der Tat fähig ist, eine solche Evolution inmitten der Natur irdisch zu tätigen, sprich leibhaftig zu vollenden. Dann, sagt das letzte YS 4.34, hat das kosmische SELBST seinen innersten Sinn und äußersten Zweck erfüllt (puruṣa-artha). Der puruṣa ist sich nun, kraft der Realisation der yogischen Gegen-Strömung der Freiheit (pratiprasavah kaivalyam) in der ihm eigenen Weite seiner Leerheit, die alle materiellen Eigenschaften der NATUR übersteigt (śūnyānām-gunānām), inmitten der Natur selbst ansichtig geworden. Damit leuchtet auch die wesentliche Form des (kosmischen) SELBST inmitten der NATUR auf (svarūpa-pratișthā), d. h. jene Kraft (citi-śaktir), die der NATUR selbst innewohnt und es dem sehenden Prinzip (purușa) erlaubt, sich inmitten der Natur selbst vernehmen zu können. Das letzte Wort des letzten Sūtra lautet „iti“. Man kann es übersetzen mit „so mag es enden“, „so ist es“, „,so sei es“ (vgl. Bäumer 2007, S. 187-192).

\section{Literatur}

Aurobindo, Sri (1992): The Synthesis of Yoga (9. Aufl.). Pondicherry.

Bäumer, Bettina (Hg.) (2007): Patañjali. Die Wurzeln des Yoga (12. Aufl.). Bern.

Deleuze, Gilles/Guattari, Felix (1974): Anti-Ödipus. Kapitalismus und Schizophrenie. Frankfurt/M.

Desikachar, T. K. V. (1980): Religiousness in Yoga. Lectures on Theory and Practice. Edited by Mary Louise Skelton/John Ross Carter. Boston.

Spinoza, Baruch de (2007): Ethik in geometrischer Ordnung dargestellt. Hamburg.

White, David G. (2012): Yoga in Practice. Princeton. 